\title{
Qualitative Study on Pile-up Effect on Hardness Test by Nano-Indentation
}

\author{
S. Sivaram, J.A.S.C. Jayasinghe and C.S. Bandara
}

\begin{abstract}
Hardness is a material parameter that enables it to resist plastic deformation, usually measured by the permanent depth of indentation. The hardness test can be carried out in macro $\left(10^{-3}\right)$, micro $\left(10^{-6}\right)$, and nano $\left(10^{-9}\right)$ levels according to the specimen size. To obtain experimental macro hardness numbers, there are several methods available such as Brinell, Vickers, and Rockwell hardness tests, etc. This study focuses on nanoindentation by numerical simulation, which leads to identifying the hardness properties of micro and nano-scale objects such as thin films and integrated circuits (IC), etc. The pile-up or sink-in effects can be observed in the nanoindentation test, which depends on the strain hardening coefficient of the target specimen. This pile-up or sink-in effect cannot be neglected in the nano-level as it obtains a considerable change of volume relative to the specimen dimension. There are no direct relationships between the pile-up or sink-in effects and mechanical properties. The objective of this study is to understand the behaviour of the pile-up effect on nanoindentation and obtain correlations between the pile-up effect and some material parameters using 2D and 3D numerical simulations.
\end{abstract}

Keywords: Mechanical properties; Nano-indentation; Nonlinear numerical simulation; Loaddisplacement curve; Pile-up.

\section{Introduction}

The nanoindentation test is an experimental method used to characterize materials at the nano and micro scale. During a nanoindentation test, a diamond indenter tip is pressed into a material up to a specified force or depth and then withdrawn. As the test is performed, the indentation force and depth are recorded, and results are generally presented in the form of a load-displacement curve. This load-displacement curve may be used to determine material properties and characteristics (Oliver et al. [13] \& Moore et al. [12]).

Some factors such as pile-up/sink-in, surface roughness, thermal drift, tip rounding, initial penetration depth, and residual stress may be affected when the indentation test is in action (Sullivan [18] \& Alaboodi et al. [2]). Therefore, serious attention should be given to the above factors when conducting the indentation test.

Nanoindentation has become popular with nanotechnology's exponential growth, leading to detailed studies and investigations measuring the hardness of certain materials. Nanoindentation is widely used in biomechanics, nanostructure mechanical characterization, and phase transition induced by pressure. An indenter with a geometry that has a proven high precision is used for the nanoindentation test. The load and displacement are recorded during the process. The indenter-generated displacements can be used to plot a load-displacement curve that can be used to extract the specimen's mechanical properties (Sivaram et al. [16] \& Bhattacharyya et al. [4]).

This pile-up or sink-in effect cannot be neglected at the Nano level as it obtains a considerable change of volume relative to the specimen dimensions. Thus, it would considerably change the exact hardness number (Bhattacharyya [4] \& Gale et al. [9]). The amount of pile-up and sink-in at the Nano indentation has a strong correlation between material parameters and the residual stress of the materials.

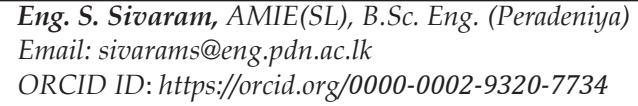

Eng. S. Sivaram, AMIE(SL), B.Sc. Eng. (Peradeniya)

Email: sivarams@eng.pdn.ac.lk

ORCID ID: https://orcid.org/0000-0002-9320-7734

Eng. (Dr.) J.A.S.C. Jayasinghe, AMIE(SL), B.Sc. Eng. (Peradeniya),M.Eng. (AIT), D. Eng. (Tokyo), Senior Lecturer, Department of Civil Engineering, Faculty of Engineering, University of Peradeniya.

Email: supunj@eng.pdn.ac.lk

ORCID ID: https://orcid.org/0000-0003-1054-9358

Eng. (Dr.) C.S. Bandara, C.Eng., MIE(SL), B.Sc. Eng. (Peradeniya), M.Sc. Eng. (Peradeniya), PhD. (Peradeniya), Senior Lecturer, Department of Civil Engineering, Faculty of Engineering, University of Peradeniya.

Email: csbandara@eng.pdn.ac.lk

ORCID ID: https://orcid.org/0000-0003-4141-8983 
Sink-in occurs in harder materials whereas pileup is common in soft materials (Oliver et al. [5] \& Zhao et al. [20]). High tensile residual stress can cause sink-in, and high compressive residual stress can cause a pile-up on the test specimen (Chen et al. [8]). An essential factor in examining the results is the type of tip used in the indentation (Cabibbo et al. [7]). Berkovich indenters are used as one of the most common types of indenter tips. Berkovich tip is faceted in the form of a three-sided pyramid, as shown in Figure 1.

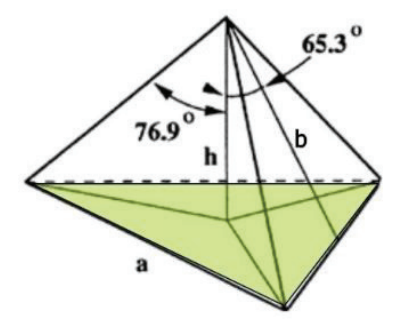

Figure 1 - Berkovich Indenter

In this study, it is focused on developing a Nano-Scale Finite Element (FE) model for indentation and to establish relationships between pile-up heights obtained from finite element modelling and material parameters.

\section{Methodology}

Figure 2 shows the general steps of the methodology of this study.

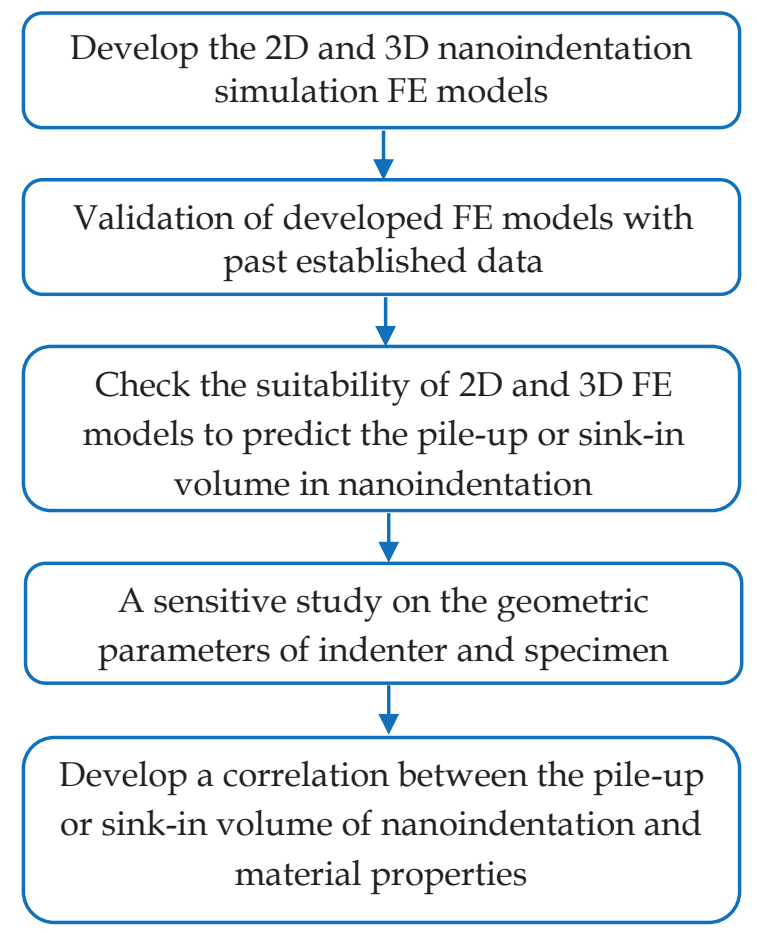

Figure 2 - Flow Chart for Steps of the Methodology

\subsection{Development of Indentation Simulation FE Models}

There are two main types of FE models used to simulate the hardness test in this research. They are 2D axisymmetric and 3D solid models. Reduced integration and hourglass control elements are used for this simulation with Von Mises plasticity model and contact theory (Lichinchi et al. [11] \& Sagadevan et al. [14]). In 2D modelling, considering the symmetry of geometry, loading, and boundary condition, half of the specimen is analyzed with proper boundary conditions (Von Mises [19]). The computational time can be reduced drastically by replacing deformable indenter by rigid indenter (Bolshakov et al. [6] \& Belytschkoet al. [3]). The number of degrees of freedom depends on the number of nodes of the system. Deformable indenter has many degrees of freedom to control the behaviour of it, while they are significantly less in the rigid indenter.

When comparing 2D and 3D rigid indenter's degrees of freedom, 3D rigid indenter has only six degrees of freedom, three translational and three rotational degrees of freedom, but 2D rigid indenter has only three degrees of freedom such as two translational and one rotational degree of freedom, at the reference point to control the behaviour (Sivaram et al. [16] \& Sivaram et al. [17]).

Furthermore, computation time can also be reduced by assigning coarser mesh away from the indentation area. Considering that, in the simulations, a refined mesh is used near the contact area, and a coarser mesh is used in other parts of the specimens (Figure 3). In 2D and 3D modelling, rigid cone and rigid Berkovich indenters are used for indentation simulations. Figure 3 and Figure 4 show the modelled 2D and 3D simulations. The friction coefficient and interaction between indenter and specimens are taken as 0.15 , and the Poisson's ratio is taken as 0.31 (Sivaram et al. [15]).

\subsection{Validation of 2D \& 3D Indentation}

Validation should be done using past studies to ensure the accuracy of the FE simulation results. The study done by Bolshakov et al. [5] is used to validate the FE models. In this study, two different finite element nanoindentation models (2D and 3D) are investigated using the commercial finite element analysis program ABAQUS (ABAQUS/ CAE [1]). 
The first model is a $2 \mathrm{D}$ axisymmetric model with a conical indenter. This type of model is commonly used in research and allows for relatively quick computational time. The second model is a $3 \mathrm{D}$ finite element model with a Berkovich indenter tip. This model is simulated for comparing and obtaining the same results, which are obtained by the 2D cone model (first model) (Bolshakov et al. [5]). Those results are also used to confirm the suitability of the finite element mesh in the 3D specimen (second model). Even though the indenter types are different in 2D and 3D models, it gives almost similar output results (Kralik et al. [10] and Bolshakov et al. [5]).

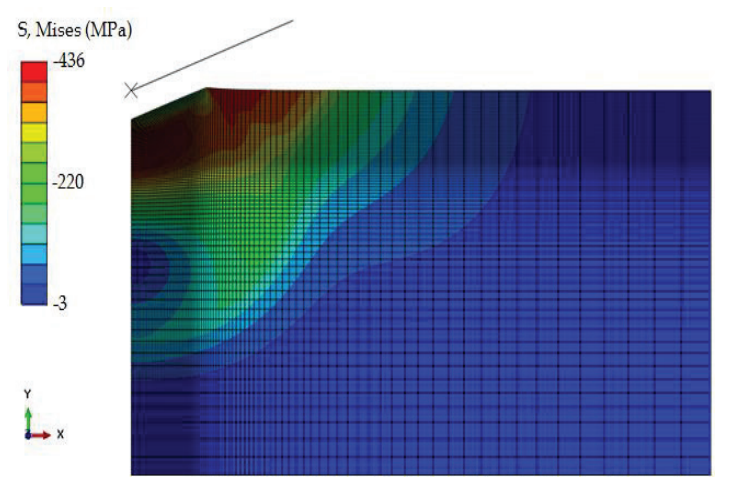

Figure 3 - 2D Cone Indentation

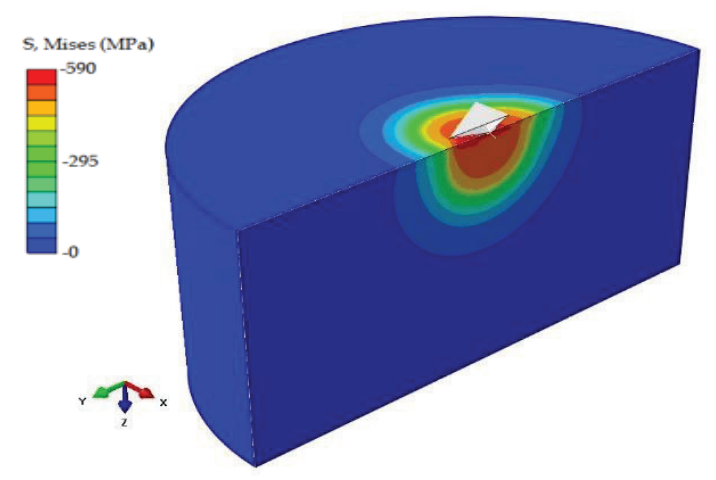

Figure 4 - 3D Berkovich Indentation

The commonly used Berkovich indenter with its three-sided pyramidal geometry should be modelled and analyzed as a three-dimensional (3D) contact problem in finite element simulations. However, the more accessible approach in the finite element simulation is using as an axisymmetric indentation model, which uses a conical shaped indenter as an equivalent to the Berkovich indenter. The main reason for this approximation is that the $3 \mathrm{D}$ contact problem requires a large capacity of computational resources and usually takes a long time to complete, especially when using a finer $3 \mathrm{D}$ mesh for accurate results. To overcome these challenges, a $70.3^{\circ}$ half-angle conical indenter is often used as a substitute for modelling the Berkovich tip, on the basis that it provides the same indentation depth to the projected area ratio of a Berkovich indenter (Bolshakov et al. [5]). By using a conical indenter, the finite element analysis can be simplified by using a 2D axisymmetric model, which allows for quicker simulations using fewer computer resources (Mooreet al. [12] and Bolshakov et al. [5]). Here, a specimen represented by a cylinder $700,000 \mathrm{~nm}$ high and $700,000 \mathrm{~nm}$ in radius was used for the simulations. Aluminum alloy 8009 is used as the material. Experimental results and results of the simulation in the literature (Bolshakov et al. [5]) are compared with our developed model. The comparison is shown in Figure 5. This proves that a $2 \mathrm{D}$ validated model can replace a 3D simulation model to simulate comfortably with an acceptable range of results.

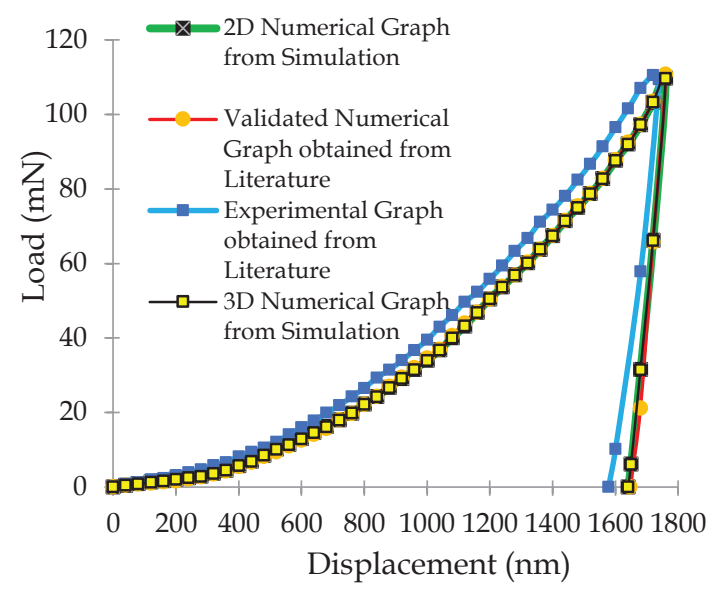

Figure 5 - The Comparison of 2D and 3D Numerical Simulations with Experimental Load-Displacement Curves

\section{Sensitivity Study}

The main objective of the sensitive study is to understand the effects of the specimen's dimensions in the load-displacement curve. Material properties with Young's modulus $(E)$ 82.1 GPa, yield strength $\left(\sigma_{y}\right) 353.1 \mathrm{MPa}$, Poisson's ratio $(v) 0.31$ of the aluminum alloy 8009 (Bolshakov et al. [5]) are used. The indentation depth of the specimen is fixed at $1600 \mathrm{~nm}$. Sensitivity studies are carried out by changing specimen depth and radius. Figure 6 expresses that there is a convergence limit for the dimensions of the specimen. It can be used to select suitable specimen dimensions for a particular loading or indentation depth value, which can reduce the number of meshes in the specimen. Consequently, that leads to reduce computational time. 
Figure 7 describes the increment of the radius of the specimen for a particular indentation; the maximum indentation load increases and converges to a particular value (nearly 110 $\mathrm{mN}$ ). From Figure 7, it can be seen that the convergence of the specimen radius achieves at $50 \mu \mathrm{m}$.

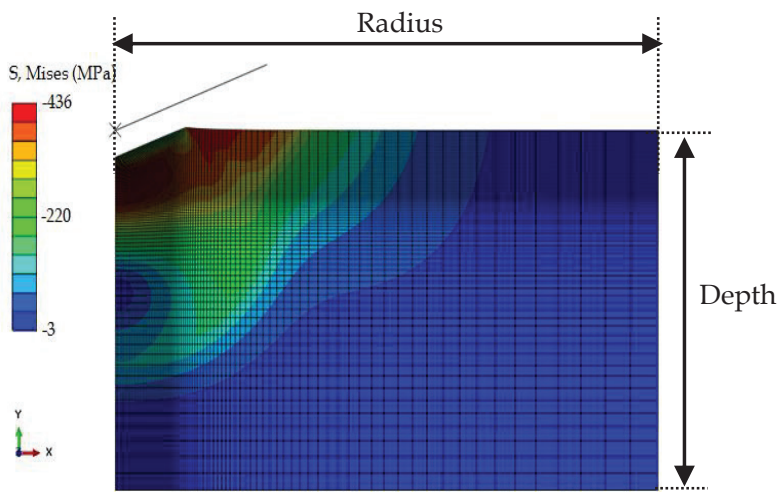

Figure 6 - Specimen Dimensions Used for the Sensitivity Study

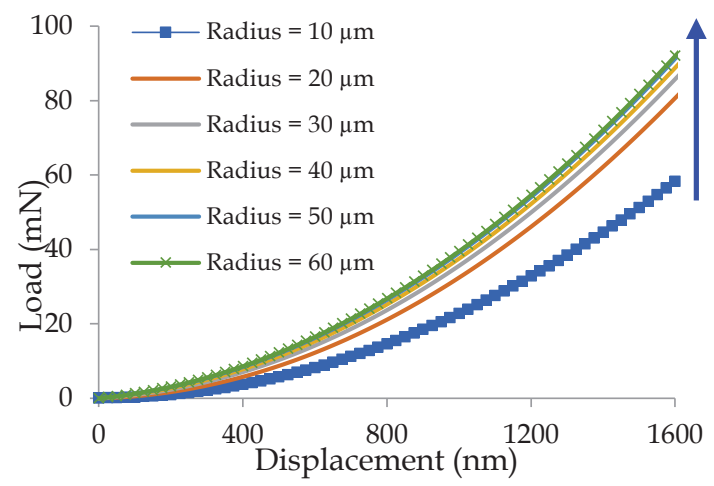

Figure 7 - The Loading Curves of 2D Cone Indentations for Different Types of Specimen Radius with Fixed Specimen depth $(100 \mu \mathrm{m})$

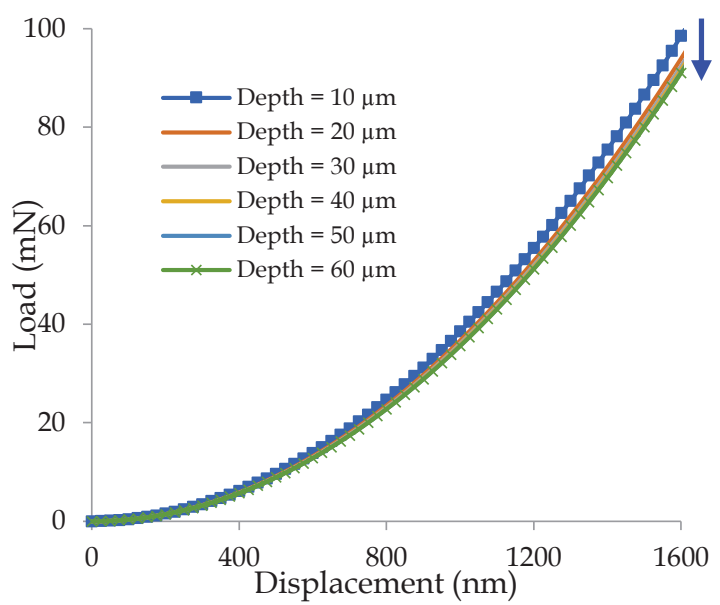

Figure 8 - The Loading Curves of 2D Cone Indentations for Different Types of Specimen Depths with a Fixed Specimen Radius (100 $\mu \mathrm{m})$

In Figure 8, it is identified with the increase of the specimen depth, and the maximum load is decreasing and converging into a particular specific value (nearly $110 \mathrm{mN}$ ). It can be seen that the convergence of the specimen depth achieves at $50 \mu \mathrm{m}$.

\section{Behaviour of the Pile-Up Effect with Indentation Depth}

Simulations are done by varying the indentation depths from $100 \mathrm{~nm}$ to $1000 \mathrm{~nm}$ to identify the pile-up effect on the specimen. Thus, the aluminum alloy 8009 (Bolshakov et al. [5]) is used for the simulation. The behaviour of the pile-up with indentation depths are presented in Figure 9.

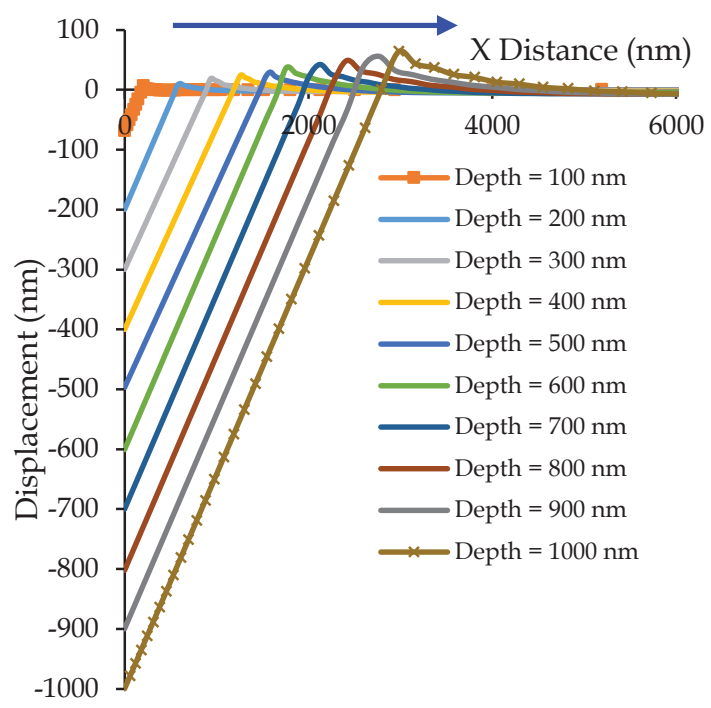

Figure 9 - Pile-Up Profile with Varying Indentation Depth

The graph between indentation depth and the maximum pile-up height for the above scenario is shown in Figure 10.

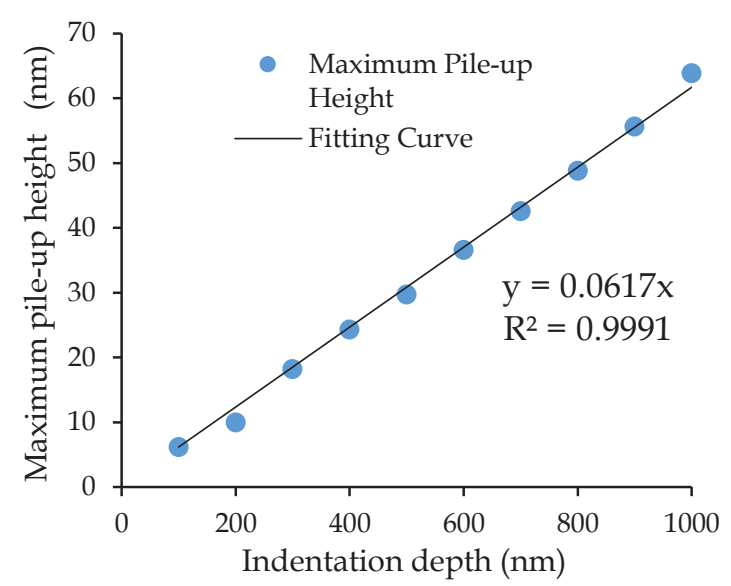

Figure 10 - The Variation of Maximum Pile-Up Height with Indentation Depth

With increasing indentation depth, the maximum pile-up height also increases, as shown in Figure 10. Consequently, when the maximum pile-up height increases, the pile-up volume also increases. The correlation between 
the maximum pile-up height and the indentation depth shows approximately a linear behaviour.

\section{Behaviour of Pile-Up Effect with Material Properties}

Simulations are done for the same specimen dimensions (same mesh characteristics) with the same indentation depth as $1000 \mathrm{~nm}$ to identify the pile-up effect on the specimen with varying material properties. In order to compare the material properties in simulations, the specimen's material properties are changed according to Table 1 .

Table 1 - Models Simulated According to the Variation of Material Properties

\begin{tabular}{|c|c|c|c|}
\hline \multirow{2}{*}{\begin{tabular}{c} 
Z \\
\cline { 2 - 4 }
\end{tabular}} & $\begin{array}{c}\text { Strain } \\
\text { hardening } \\
\text { haterial properties } \\
\text { coefficient }(n)\end{array}$ & $\begin{array}{c}\text { Yield } \\
\text { strength } \\
(\mathrm{MPa})\end{array}$ & $\begin{array}{c}\text { Young's } \\
\text { modulus } \\
(\mathrm{GPa})\end{array}$ \\
\hline 1 & Vary (0.1-0.48) & 300 & 75 \\
\hline 2 & 0.1 & Vary (75-345) & 75 \\
\hline 3 & 0.1 & 300 & Vary (50-240) \\
\hline
\end{tabular}

\subsection{Behaviour of the Strain Hardening Component $(n)$}

Strain hardening coefficient $(n)$ is a measure of how much the metal can be strengthened by strain hardening. It needs to have some ductility to be strain hardened. In simple terms, $n$ is the slope of the plastic portion of the true stress with the true strain curve when graphed on a logarithmic scale.

The selected load-displacement curves are obtained by using case 1 , which is represented in Table 1. Figure 11 shows that, for a fixed indentation depth (1000 nm), loaddisplacement curves are varying with strain hardening coefficient.

The maximum load of a fixed indentation depth is linearly increasing with the strain hardening coefficient in the load-displacement curve shown in Figure 12. For the high hardness material, the strain hardening component is high. Therefore, the required force needs to penetrate for fixed indentation depth increases with strain hardening component. The unloading curve shows the same gradient without any change. That implies that Young's modulus is not varying in this scenario.

The pile-up profiles by varying the strain hardening coefficient are shown in Figure 13.
The correlation between maximum pile-up height with strain hardening coefficient is shown in Figure 14.

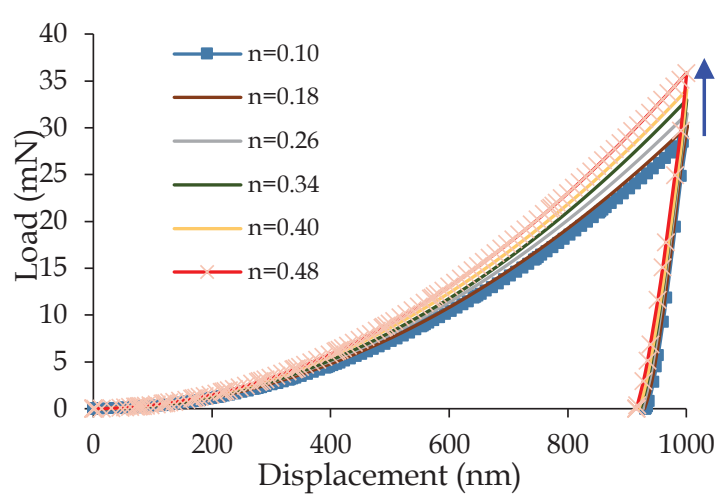

Figure 11 - Selected Load-Displacement Curves while Varying the Strain Hardening Coefficient

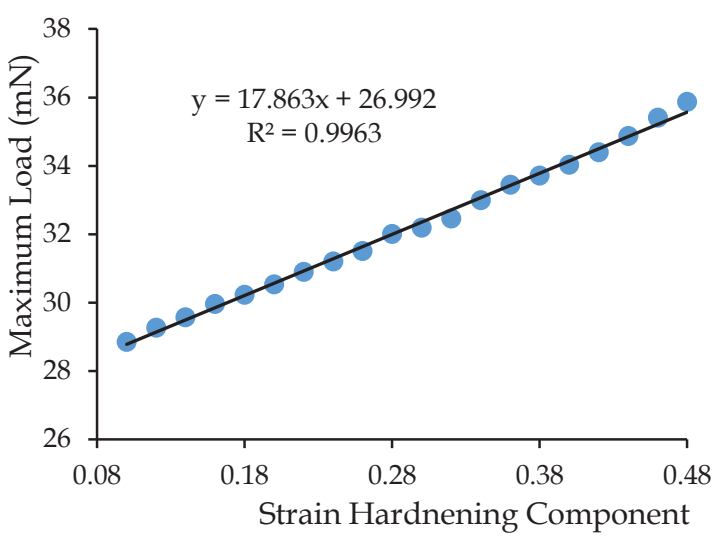

Figure 12 - The Maximum Indentation Load by Varying the Strain Hardening Coefficient

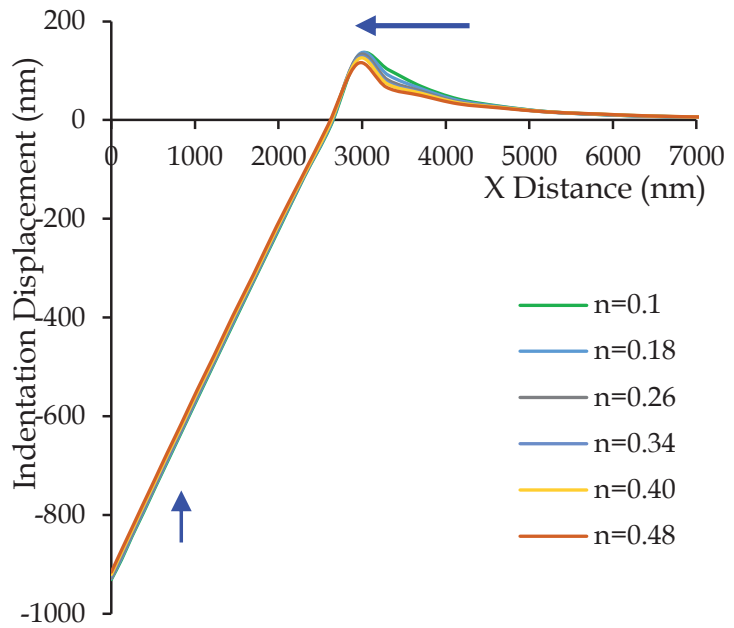

Figure 13 - Pile-Up Profiles by Varying the Strain Hardening Coefficient

As shown in Figure 14, with the increment of the strain hardening coefficient, the recovery depth of the indentation is increased. 


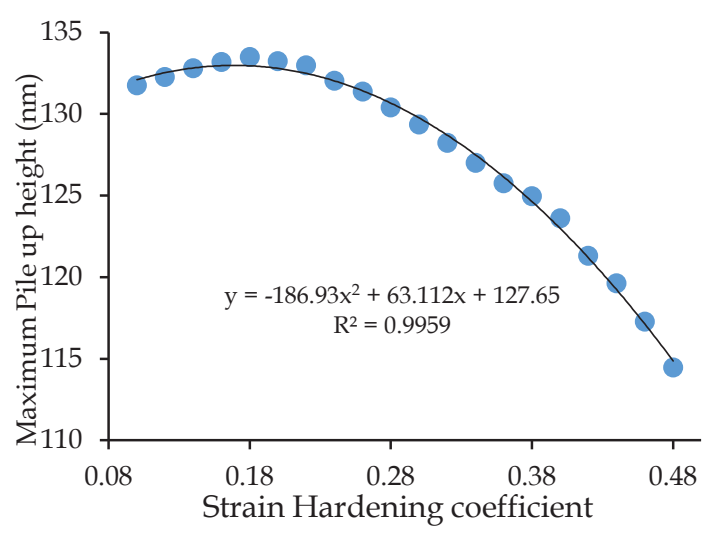

Figure 14 - The Variation of Maximum Pile-Up Height with the Strain Hardening Coefficient

\subsection{Behaviour of the Yield Strength}

The selected load-displacement curves, obtained by using case 2 (corresponding to Table 1), are presented in Figure 15. The unloading curves in Figure 15 show the same gradient without any changes in Young's modulus. As mentioned before, this implies that Young's modulus is not varying in this scenario.

Figure 16 shows that the maximum loads for a fixed indentation depth are linearly increasing with yield strength. With the increase of the yield strength, the material is getting hardened. It implies that the force needing for penetration is increasing with the yield strength for a fixed indentation depth.

The pile-up profiles by varying the yield strengths are shown in Figure 17. Correlation between maximum pile-up heights with yield strength is shown in Figure 18.

As shown in Figure 17, with the increase of the yield strength, the recovery depth of the indentation is increased. In the beginning, to control the pile-up volume, considering both strain hardening component and yield strength (Figures 13 and 17), the maximum pile-up height also increased. The shape of the outer surface of the pile-up profile declines gradually. However, from a certain point, pile-up volume starts to reduce. Similarly, the maximum pileup height is also reduced. Because of this reason, the graphs in Figure 14 and Figure 18 are obtained as a parabolic path.

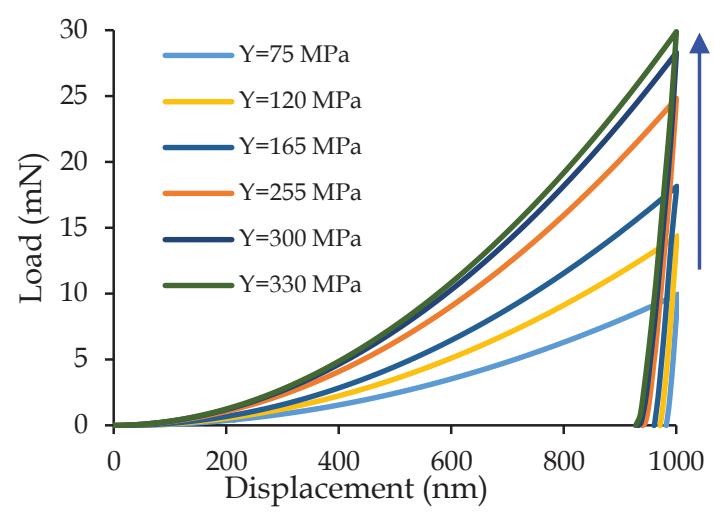

Figure 15 - Load-Displacement Curves while Varying the Yield Strength

\subsection{Behaviour of the Young's Modulus}

The selected load-displacement curves obtained using case 3 (corresponding to Table 1) are presented in Figure 19.

Figure 20 shows a variation of slopes in the unloading curves of the load-displacement curves. Considering Figure 19, it can be said that unloading slope depends on Young's modulus.

The maximum load of an indentation test increases with Young's modulus in the loaddisplacement curve, as shown in Figure 19. Because of the increment in Young's modulus, the material is getting harder, and it becomes difficult for penetrating to a certain indentation depth. The unloading curves show a variation of their slopes in the load-displacement curves.

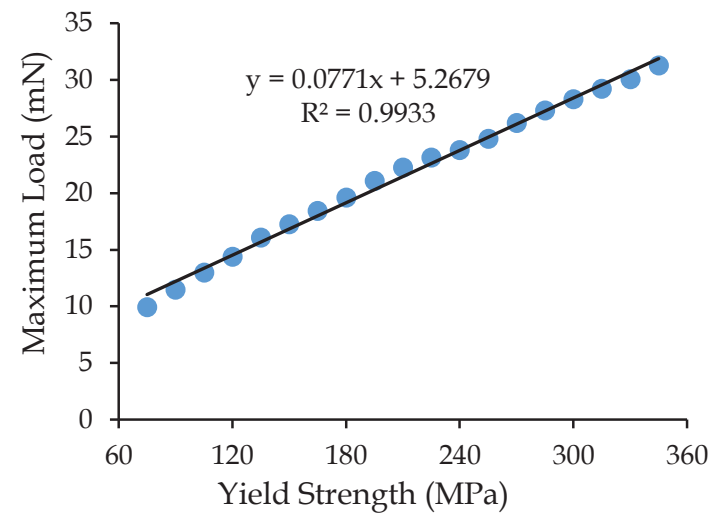

Figure 16 - The Maximum Indentation Load by Varying the Yield Strength 


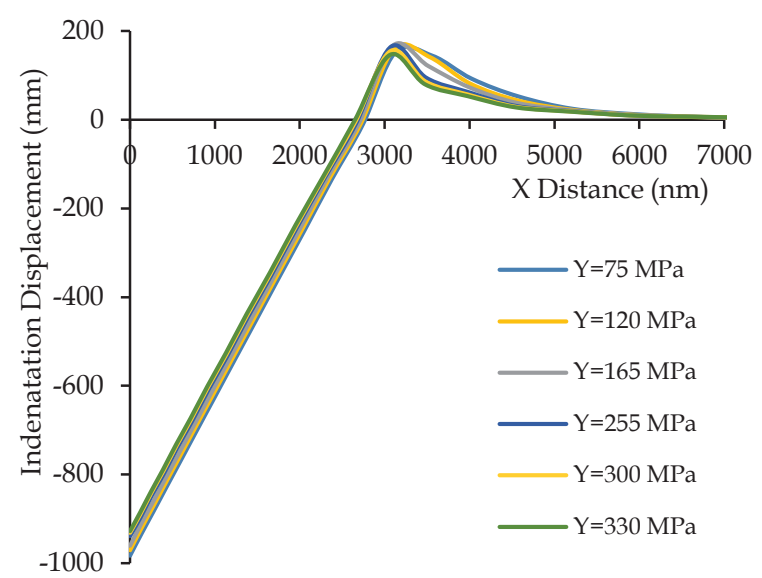

Figure 17 - Pile-Up Profiles by Varying Yield Strength

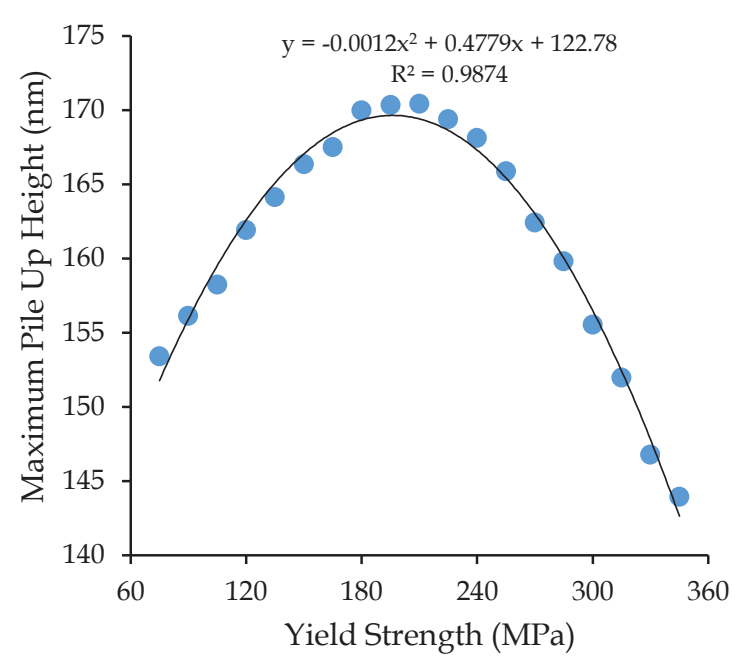

Figure 18 - The Variation of Maximum PileUp Height with Yield Strength

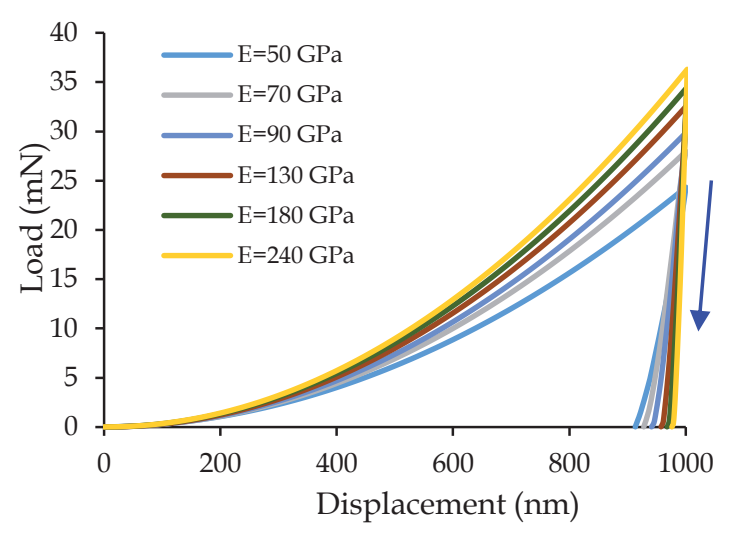

Figure 19 - Load-Displacement Curves while Varying Young's Modulus (E)

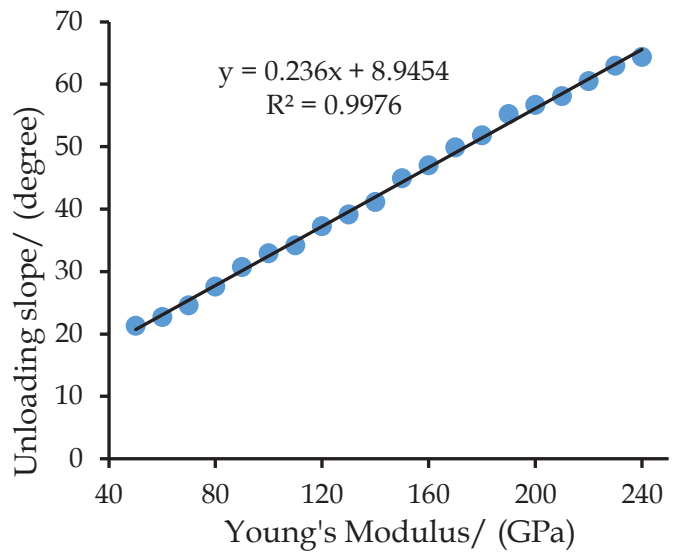

Figure 20 - The Slope of Unloading Curves with Varying Young's Modulus

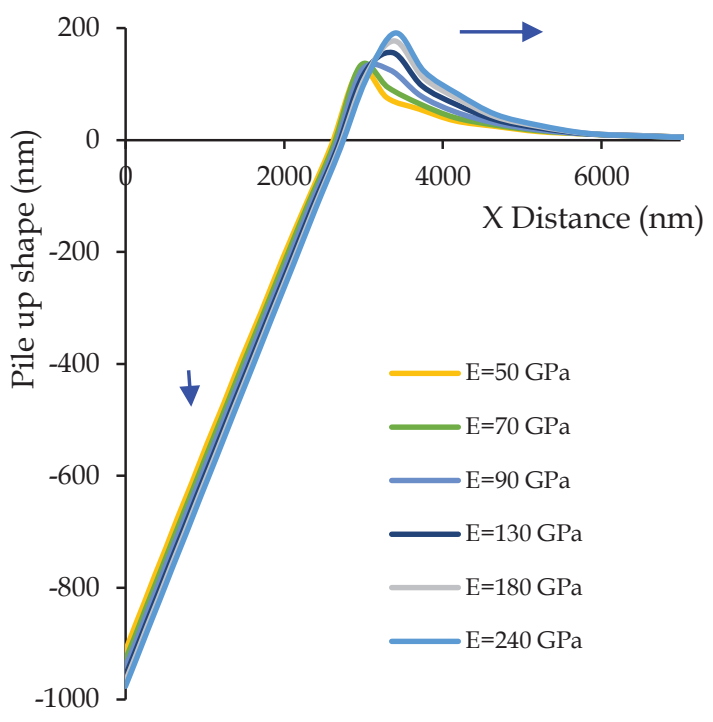

Figure 21 - Pile-Up Profiles by Varying Young's Modulus (E)

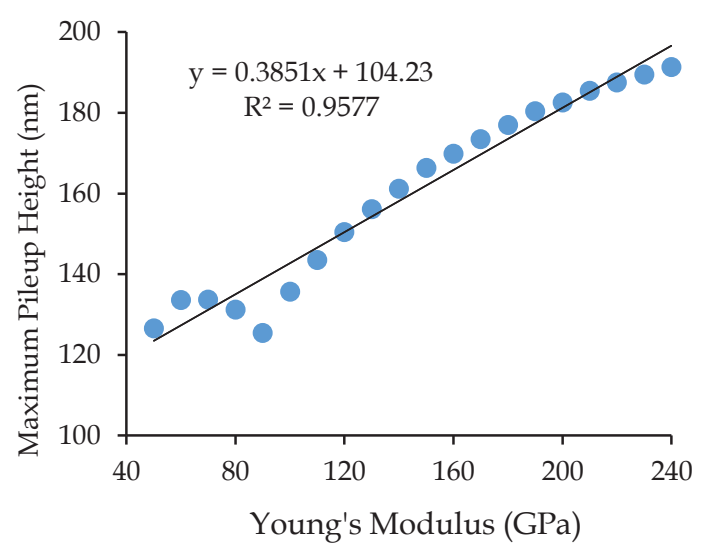

Figure 22 - The Variation of Maximum Pile-Up Height with Young's Modulus 
The slope of unloading curves shows the linear relationship with Young's modulus, as shown in Figure 20. The pile-up profiles by varying Young's modulus are shown in Figure 21, where the maximum pile-up heights with Young's modulus are shown in Figure 22. Considering Figure 22, approximately maximum pile-up height is linearly varying with Young's modulus.

\section{Conclusions}

In this study, the pile-up effect on nanoindentation using numerical simulation is carried out. The main objectives of this study are to understand the behaviour of the pile-up effect on nanoindentation by using 2D and 3D numerical simulations and also to obtain correlations between the pile-up effect and material properties. Von Mises plasticity model is used for the specimen to get the real metal behaviour for the specimens. Two-dimensional FE models with cone indenter of half-angle $70.3^{\circ}$ were used to replace the threedimensional FE models with Berkovich indenter to simplify the simulation process. The conclusions made from the results are presented as follows.

- When indentation depth increases, pile-up height shows a linear behaviour. This relationship (Figure 10) can be used to find the approximate maximum pile-up value for this selected material.

- When varying the strain hardening coefficient and yield strength, approximately linear relationships are observed with the maximum indentation load (Figures 12 and 16) for a particular indentation depth. In addition to that, good relationships are obtained for maximum pile-up height, yield strength, and Young's modulus with strain hardening component (Figures 14, 18 and 22).

- Moreover, an approximately linear relationship is developed between Young's modulus and the unloading curve slope, as shown in Figure 20.

- All the relationships obtained from this study (Figures 12, 14, 16, 18, 20 and 22) can be used as an inverse analysis to extract the material parameters for other materials, which will help to develop further studies.

\section{Acknowledgment}

University Research Grant, URG/2018/23/E of the University of Peradeniya is highly appreciated.

\section{References}

1. ABAQUS/CAE User's Manual Version 6.14. (2016) Providence: ABAQUS, Inc.

2. Alaboodi, A. S., \& Hussain, Z. (2017), "Finite Element Modeling of Nanoindentation Technique to Characterize Thin Film Coatings", Engineering Sciences, pp.1-9.

3. Belytschko, T., Ong, J. S. J., Liu, W. K., \& Kennedy, J. M. (1984),"Hourglass Control in Linear and Nonlinear Problems", Computer Methods in Applied Mechanics and Engineering, Vol. 43, Issue 3, pp. 251-276.

4. Bhattacharyya, A. S. (2010), "Tribological and Surface Engineering Properties of SiCN Thin Films", Proc. of the 3rd International Conference on Advances in Mechanical Engineering.

5. Bolshakov, A., Oliver, W. C., \& Pharr, G. M. (1996) "Influences of Stress on the Measurement of Mechanical Properties Using Nanoindentation", Journal of Materials Research, Vol.11(3), pp.760-768.

6. Bolshakov, A., \& Pharr, G. M. (1998), "Influences of Pile-Up on the Measurement of Mechanical Properties by Load and Depth Sensing Indentation Techniques", Journal of Materials Research, Vol. 13, pp. 1049-1058.

7. Cabibbo, M., Ciccarelli, D., \& Spigarelli, S. (2013), "Nano-Indentation Hardness Measurement in Piling Up SiO2 Coating", 2nd European Conference on Nano Films, Vol.40, pp.100-112.

8. Chen, X., Yan, J., \& Karlsson, A. M. (2006), "On the Determination of Residual Stress and Mechanical Properties by Indentation", Materials Science and Engineering: A, Vol.416(1-2), pp.139-149.

9. Gale, J. D., \&Achuthan, A. (2014), "The Effect of Work-Hardening and Pile-Up on Nanoindentation Measurements",Journal of Materials Science, Vol.49(14), pp.5066-5075.

10. Kralik, V., \& Nemecek, J. (2014), "Comparing Nanoindentation Techniques for Local Mechanical Quantification of Aluminum Alloy", Materials Science and Engineering, Vol.618, pp.118128.

11. Lichinchi, M., Lenardi, C., Haupt, J. \& Vitali, R. (1998), "Simulation of Berkovich Nanoindentation Experiments on Thin Films Using Finite Element Method", This Solid Films, Vol.312, pp.240-248.

12. Moore, S. W., Manzari, M. T., \& Shen, Y. L. (2010), "Nano-Indentation in Elastoplastic Materials: Insights from Numerical simulations", International Journal of Smart and Nano Materials, Vol.1:2, pp.95-114. 
13. Oliver, W. C., \& Pharr, G. M. (2004), "Measurement of Hardness and Elastic Modulus by Instrumented Indentation: Advances in Understanding and Refinements to Methodology", Journal of Materials Research, Vol. 19, pp. 3-20.

14. Sagadevan, S., \& Murugasen, P. (2014), "Novel Analysis on the Influence of Tip Radius and Shape of the Nanoindenter on the Hardness of Materials", 3rd International Conference on Materials Processing and Characterisation, Procedia Materials Science, Vol.6, pp.1871-1878.

15. Sivaram, S., Sutharsan, K., Jayasinghe, J. A. S. C., \& Bandara, C. S. (2017), "Development of Indentation Testing Method for Evaluation of Mechanical Properties of Steel Reinforcement", $8^{\text {th }}$ International Conference on Structural and Construction Management, pp.85-93.

16. Sivaram, S., Jayasinghe, J. A. S. C., \& Bandara, C. S. (2018), "Numerical Simulation of Indentation Test Method to Evaluate Residual Strain of Metals", Advances in Civil and Environmental Engineering Practices for Sustainable Development, pp.302-308.

17. Sivaram, S., Jayasinghe, J. A. S. C., \& Bandara, C. S. (2018), "Prediction of Residual Strain of Steel Using Brinell Hardness Number: Development and Application to Old Bridge Structures in SriLanka", Society of Structural Engineers Sri Lanka, pp.79-85.

18. Sullivan, M. (2015), "Measuring, Evaluating and Describing Pile-Up and Sink-In During Nanoindentation of Thin Films on Substrates".

19. Von Mises, R. (1913), "Mechanics of Solid Bodies in a Plastically Dformable State", MathematicalPhysics,Vol. 1, pp. 582-592.

20. Zhao, M., Chen, X., Yan, J., \& Karlsson, A. M. (2006), "Determination of Uniaxial Residual Stress and Mechanical Properties by Instrumented Indentation", Acta Materialia, Vol. 54(10). 